\title{
Adverse Effects of Aggressive Blood Pressure Control in Patients with Intracerebral Hemorrhage
}

\author{
Panagiotis Mastorakos, $\mathrm{MD}^{1}$, Kenneth C. Liu, MD ${ }^{1,2}$, and Andrew Schomer, $\mathrm{MD}^{3}$ \\ ${ }^{1}$ Department of Neurological Surgery, University of Virginia Health System, Charlottesville, VA, USA, \\ ${ }^{2}$ Department of Radiology \& Medical Imaging, University of Virginia Health System, Charlottesville, VA, USA, \\ ${ }^{3}$ Department of Neurology, University of Virginia Health System, Charlottesville, VA, USA
}

Background: Medical management of patients presenting with spontaneous intracerebral hemorrhage (ICH) is focused on blood pressure (BP) management. However, the BP goal to prevent $\mathrm{ICH}$ expansion remains controversial. Recent clinical trials have suggested that aggressive BP control is safe but may not have the previously thought benefits.

Case Report: We present an example of aggressive BP control in the setting of hypertensive $\mathrm{ICH}$, in accordance to previously established protocols. This resulted in adverse effects in the form of acute kidney injury and watershed infarcts, which impeded the patients' recovery and prolonged his hospitalization.

Conclusions: Hypertensive individuals have altered cerebral autoregulation curves shifted to the right and require higher arterial pressures to maintain adequate cerebral blood flow. Hence, aggressive BP reduction may result in cerebral hypoperfusion as well as other forms of end-organ damage.

J Neurocrit Care 2017;10(1):36-40

Key words: Intracerebral hemorrhage; Blood pressure; Hypoperfusion; Cerebral infarction

\author{
Received December 31, 2016 \\ Revised February 8, 2017 \\ Accepted February 9, 2017 \\ Corresponding Author: \\ Panagiotis Mastorakos, MD \\ Box 800212, Department of \\ Neurological Surgery, University of \\ Virginia Health System Charlottesville, \\ Virginia 22908, USA \\ Tel: +1-434-924-8129 \\ Fax: +1-434-243-6726 \\ E-mail:pm5mt@virginia.edu
}

Copyright $\odot 2017$ The Korean Neurocritical Care Society

\section{INTRODUCTION}

The primary goal in patients presenting with intracerebral hemorrhage $(\mathrm{ICH})$ is to prevent hemorrhage expansion and the resultant deterioration. Reversal of anticoagulation, and aggressive blood pressure control are considered the cornerstones of ICH management. I ICH expansion occurs primarily within 6 hours from initial diagnosis but for $37 \%$ of patients it may continue up to 24 hours; an initial systolic blood pressure (SBP) of $\geq 200 \mathrm{mmHg}$ is associated with hematoma expansion and increased mortality. ${ }^{2}$ A number of studies demonstrate a correlation between higher initial blood pressures and worse outcome. ${ }^{2}$ This, in addition to the lack of supporting evidence regarding the risk of ischemia in the region surrounding the hemorrhage, have led to increasingly lower SBP or mean arterial pressure (MAP) goals in ICH management. ${ }^{3}$ Recent clinical trials have suggested that aggressive blood pressure control may not have the previously thought benefits. ${ }^{1,4}$

\section{CASE REPORT}

A 40 year-old male with no known past medical history, but suspected undiagnosed chronic hypertension, presented with sudden onset of headache associated with bilateral blurry vision,

(cc) This is an Open Access article distributed under the terms of the Creative Commons Attribution Non-Commercial License (http://creativecommons.org/licenses/by$\mathrm{nc} / 4.0$ ) which permits unrestricted non-commercial use, distribution, and reproduction in any medium, provided the original work is properly cited. 

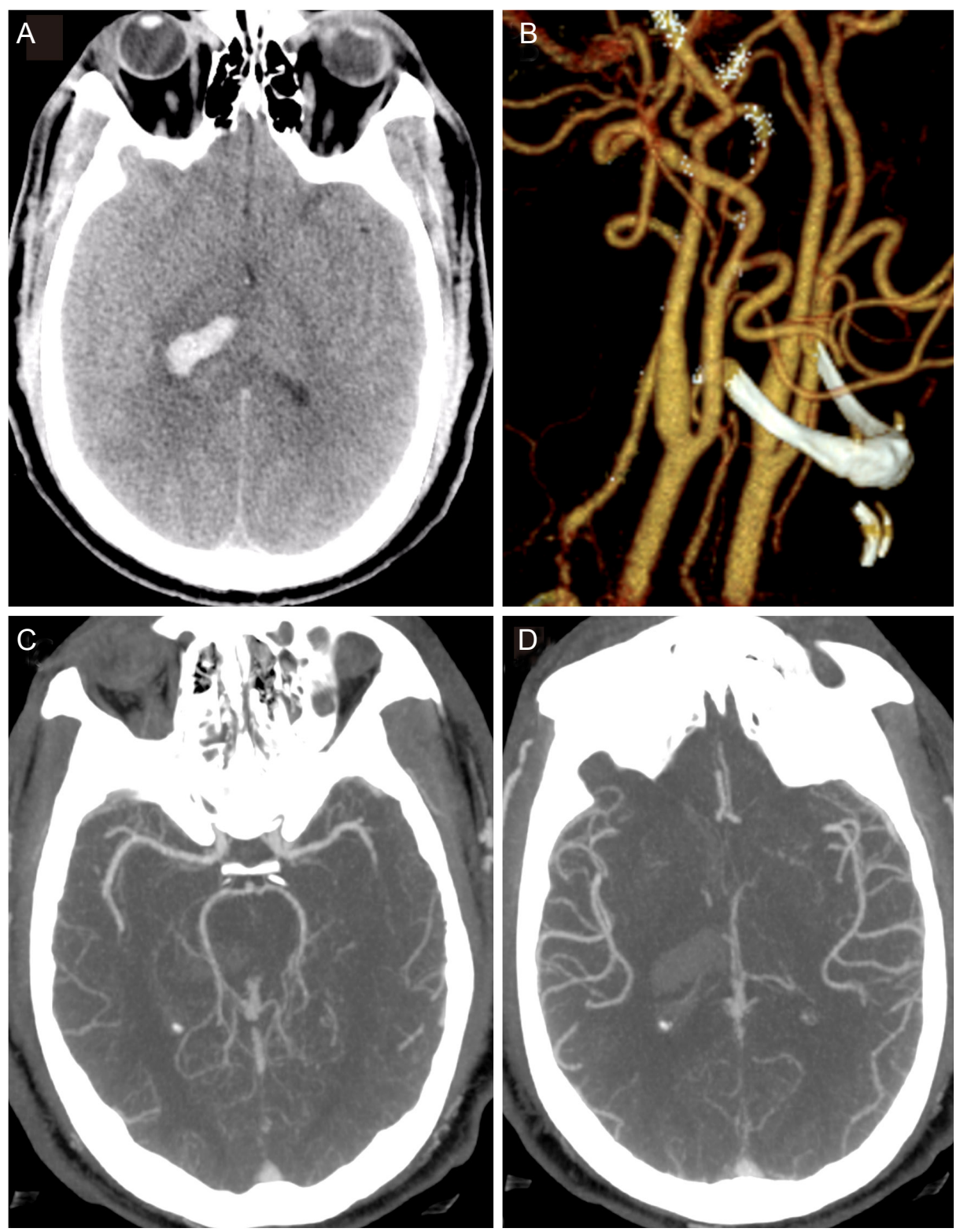

Figure 1. (A) CT head at presentation demonstrating score 1 thalamic ICH; (B) 3D reconstitution of bilateral carotid arteries demonstrating patent vasculature and no evidence of significant stenosis; (C), (D) CT angiogram demonstrating patent intracranial vasculature at presentation.

bilateral perioral tingling and left sided weakness 9 hours prior to admission. Neurologic exam was significant for right gaze preference and a left hemiparesis. Computer tomography (CT) of the head demonstrated a right thalamic $\mathrm{ICH}$, which was graded as ICH score 1 on admission (GCS 15, volume < $30 \mathrm{cc}$, positive IVH, supratentorial origin, age < 80 years; Fig. $1 \mathrm{~A}) .^{5} \mathrm{CT}$ angiogram demonstrated no vascular abnormality and no evidence of carotid stenosis (Fig. 1B-D). Chest X-ray demonstrated evidence of cardiomegaly suggestive of chronic hypertension. His initial blood pressure was 220/150 mmHg. He was placed on nicardipine and nitroprusside drip targeting SBP less than $140 \mathrm{mmHg}$, which resulted in a reduction of his $\mathrm{BP}$ to $120 / 70 \mathrm{mmHg}$ within 20 minutes. However, there was no evidence of cardiogenic shock with troponin $0.3 \mathrm{ng} / \mathrm{mL}$ and electrocardiogram only suggestive of left ventricular hypertrophy. Thus, SBP was subsequently kept between 120 to $140 \mathrm{mmHg}$ for $24 \mathrm{~h}$ and relaxed to 

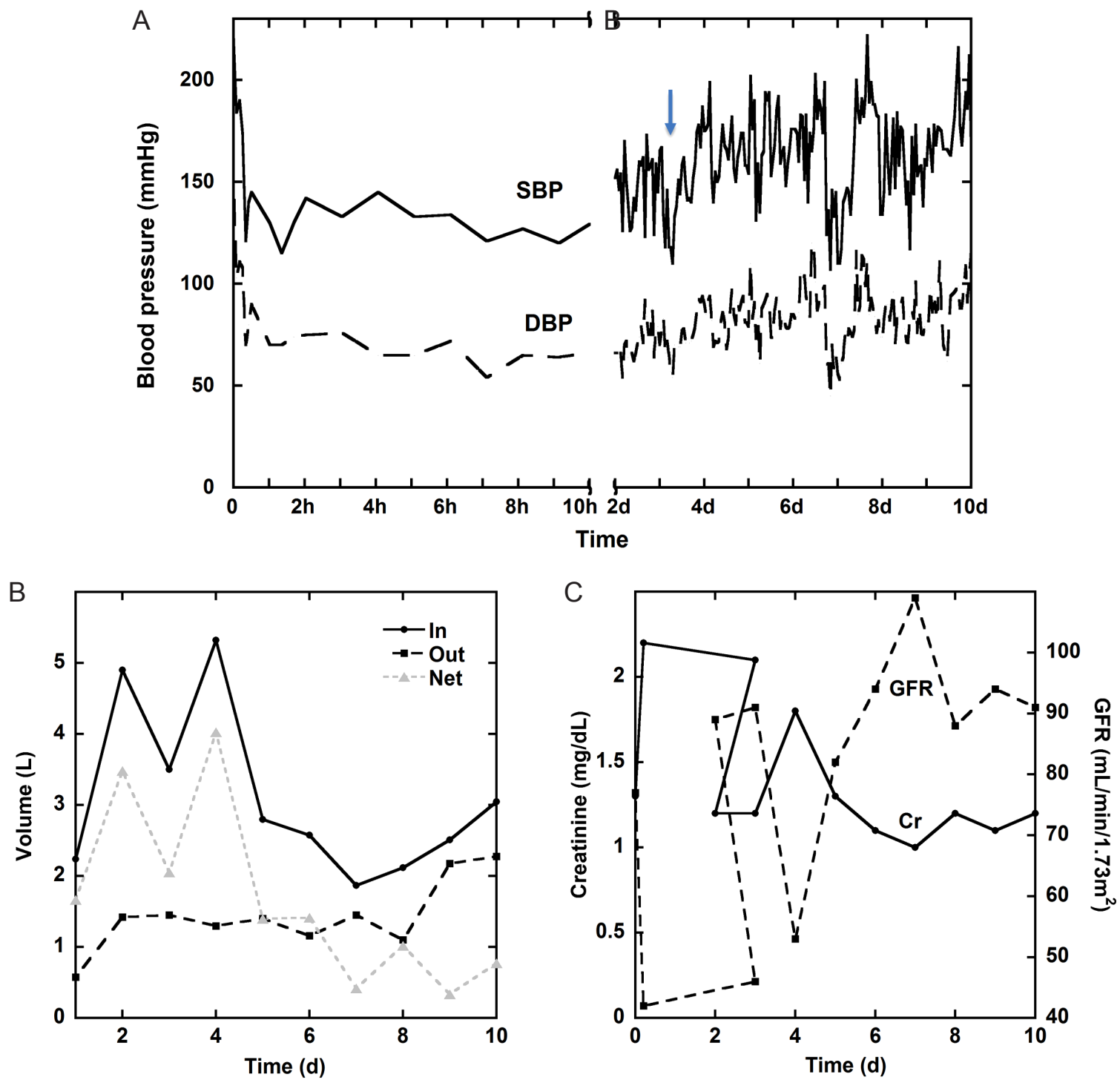

Figure 2. (A) Systolic and diastolic blood pressure during the first 10d of admission, the arrow indicates the time of detection of the stroke; (B) Daily volume balance, including in, out and net, during admission; (C) Daily creatinine ( $\mathrm{Cr}$ ) and glomerular filtration rate (GFR) during admission.

\section{SBP $<160 \mathrm{mmHg}$ the next day (Fig. 2A).}

He continued to require maximum nicardipine drip rate in addition to oral medications till post bleed day (PBD) 3. Given the high blood pressure at presentation and the high antihypertensive requirements we completed a work up for secondary hypertension. A renal ultrasound demonstrated no renal artery stenosis, plasma metanephrines were $<0.20 \mathrm{nmol} / \mathrm{L}$ and the aldosterone-ration was 1.4, thus providing no evidence of secondary hypertension.

Within 5 hours from admission, the patient developed acute kidney injury (AKI) with an increased creatinine from 1.3 to 2.2 and low urine output to $0.3 \mathrm{~mL} / \mathrm{kg} / \mathrm{hr}$. Urine electrolytes were consistent with pre-renal azotemia based on the fractional excretion of sodium (FENa $=0.3 \%$ ) below $1 \%$. The patient's AKI gradually resolved, as demonstrated by urine output, creatinine and glomerular filtration rate (GFR) trends, with fluid hydration and relaxed BP goals (Fig. 2B, C).

Approximately 10 hours after admission the patient had an acute deterioration in exam with more pronounced left sided hemiparesis and diminished level of arousal. Repeat CT head showed stable ICH and electroencephalogram demonstrated no 


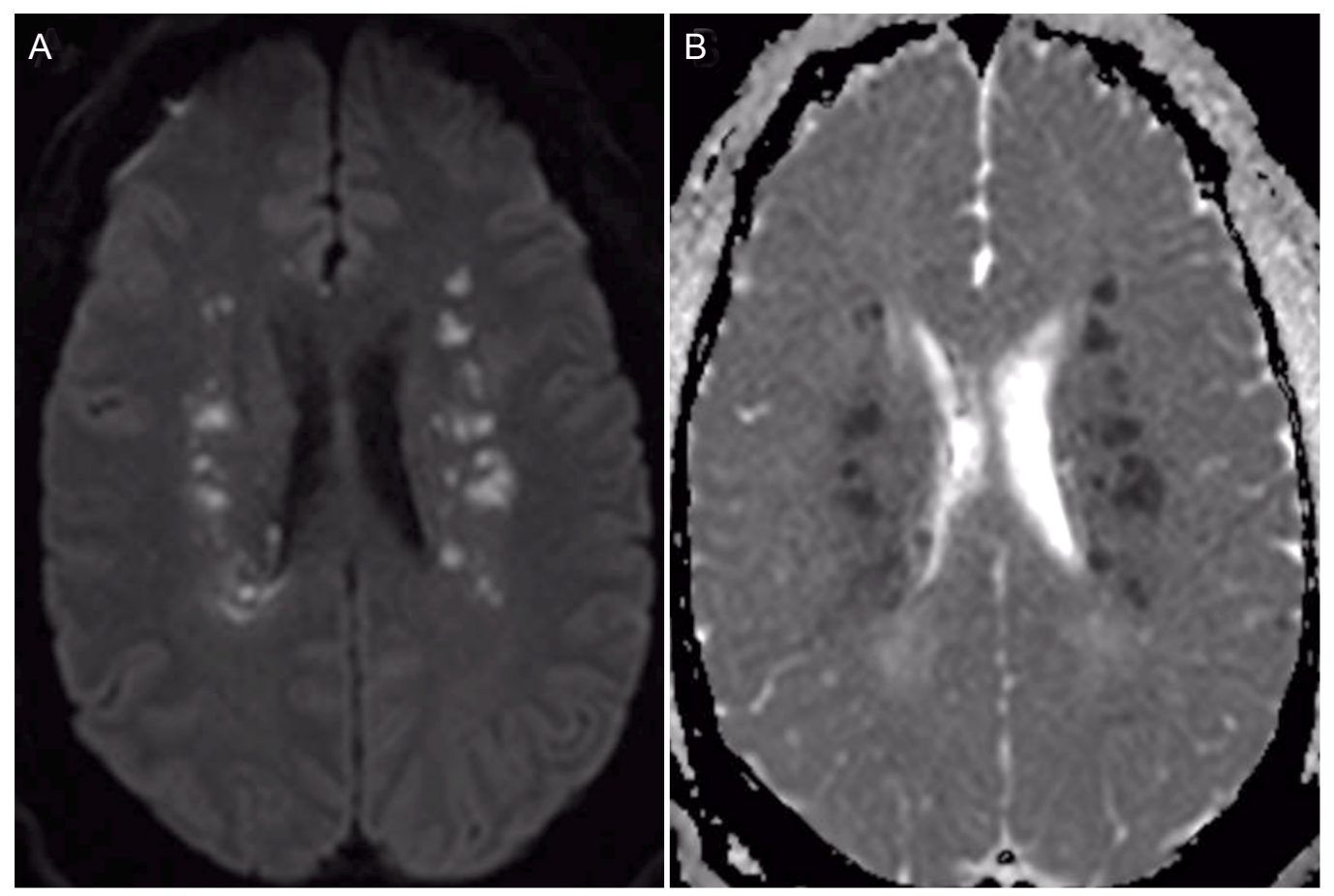

Figure 3. (A) MRI DWI and (B) MRI ADC demonstrating watershed infarcts.

evidence of seizures. Patient's exam continued to wax and wane with persistently decreased level of alertness and worsening dysarthria compared to initial presentation. On PBD 3 the patient became obtunded and required intubation for airway protection. A brain magnetic resonance image (MRI) revealed multiple areas of deep white matter infarction predominately in the frontoparietal lobes compatible with watershed infarction (Fig. 3). SBP goal was increased to less than $180 \mathrm{mmHg}$, which was achieved with an oral antihypertensive regimen. The patient's exam gradually improved, he was able to be extubated and gradually recovered following a 3-week hospital stay.

\section{DISCUSSION}

Recently, the INTERACT2 trial demonstrated that aggressive SBP lowering to a goal of less than $140 \mathrm{mmHg}$ is safe and resulted in a small yet significant improvement in clinical outcomes. ${ }^{4}$ Based on these findings the 2015 AHA guidelines suggested that acute lowering of SBP to $140 \mathrm{mmHg}$ was safe and could be an effective method to improve functional outcomes. ${ }^{1}$ Later, the ATACH-2 trial, which attempted to achieve greater therapeutic benefit by more rapidly achieving the SBP goal when comparing intensive (systolic blood-pressure target of 110 to $139 \mathrm{mmHg}$ ) or standard (140 to $179 \mathrm{mmHg}$ ) care, was terminated prematurely for futility. Apart from establishing non-superiority of aggressive BP management, the ATACH-2 trial underlined the potential risk of SBP reduction to a goal of $<140 \mathrm{mmHg}$, demonstrating in a post-hoc analysis, an increased rate of renal adverse events at 7 days in the intense BP control group as opposed to the standard group ( $9 \%$ vs $4 \%, P=0.002$ ), but no significant difference in the rates in other adverse-events. ${ }^{6}$

The ATACH-2 trial failed to show a significant benefit from aggressive SBP control, but no serious adverse events related to treatment were seen within the first 72 hours. Importantly, the guidelines for the treatment for hypertensive emergency suggest SBP reduction of $15-25 \%$ of the initial value, in order to avoid endorgan hypoperfusion. ${ }^{7}$ Hypertensive individuals have cerebral autoregulation curves shifted to the right and require higher arterial pressures to maintain adequate cerebral blood flow. Hence, aggressive SBP reduction may result in cerebral hypoperfusion. ${ }^{8}$ Moreover, excessive unintended decrease in BP in this patient population is common, ${ }^{9}$ and the Euro-STAT observational study demonstrated that in $10 \%$ of cases intravenous antihypertensive 
therapy resulted in hypotension. ${ }^{10}$

Setting a universal blood pressure goal in the $\mathrm{ICH}$ patient population may prove too difficult as cerebral autoregulation may vary widely due to the disease process itself as well as history of hypertension. Advances in neuromonitoring have provided multiple methods to determine cerebral blood flow (CBF) as well as cerebral autoregulation, including transcranial doppler, nearinfrared spectroscopy, brain tissue oxygen monitoring, laser doppler flowmetry, and thermal diffusion. Using continuous CBF monitoring may allow us to set individualized BP goals that do not dangerously decrease cerebral perfusion."

This case emphasizes that the practice of intensive SBP control in patients with $\mathrm{ICH}$ can be harmful, result in end-organ damage and contribute to prolonged hospital stay. Hopefully, with further advances in neuromonitoring, and trials targeting patient-specific blood pressure goals, we can decrease the frequency of complications associated with the treatment of hypertension in these critically ill patients.

\section{Conflicts of interest}

Panagiotis Mastorakos, MD, Kenneth C. Liu, MD, and Andrew Schomer, MD report no disclosures.

\section{REFERENCES}

1. Hemphill JC, 3rd, Greenberg SM, Anderson CS, Becker K, Bendok BR, Cushman M, et al. Guidelines for the Management of Spontaneous Intracerebral Hemorrhage: A Guideline for Healthcare Professionals From the American Heart Association/American Stroke Association. Stroke 2015;46:2032-60.
2. Dandapani BK, Suzuki S, Kelley RE, Reyes-Iglesias Y, Duncan RC. Relation between blood pressure and outcome in intracerebral hemorrhage. Stroke 1995;26:21-4.

3. Qureshi Al. The Importance of Acute Hypertensive Response in ICH. Stroke 2013;44:S67-S9.

4. Anderson CS, Heeley E, Huang Y, Wang J, Stapf C, Delcourt $\mathrm{C}$, et al. Rapid blood-pressure lowering in patients with acute intracerebral hemorrhage. N Eng/ / Med 2013;368:2355-65.

5. Hemphill JC 3rd, Bonovich DC, Besmertis L, Manley GT, Johnston SC. The ICH score: a simple, reliable grading scale for intracerebral hemorrhage. Stroke 2001;32:891-7.

6. Qureshi Al, Palesch YY, Barsan WG, Hanley DF, Hsu CY, Martin RL, et al. Intensive Blood-Pressure Lowering in Patients with Acute Cerebral Hemorrhage. N Engl / Med 2016;375:1033-43.

7. Muiesan ML, Salvetti M, Amadoro V, di Somma S, Perlini S, Semplicini A, et al. An update on hypertensive emergencies and urgencies. / Cardiovasc Med (Hagerstown) 2015;16:37282.

8. Blumenfeld JD, Laragh JH. Management of hypertensive crises: the scientific basis for treatment decisions. Am I Hypertens 2001;14:1154-67.

9. Brooks TW, Finch CK, Lobo BL, Deaton PR, Varner CF. Blood pressure management in acute hypertensive emergency. Am J Health Syst Pharm 2007;64:2579-82.

10. Katz JN, Gore JM, Amin A, Anderson FA, Dasta JF, Ferguson JJ, et al. Practice patterns, outcomes, and end-organ dysfunction for patients with acute severe hypertension: the Studying the Treatment of Acute hyperTension (STAT) registry. Am Heart I 2009;158:599-606 e1.

11. Donnelly J, Budohoski KP, Smielewski P, Czosnyka M. Regulation of the cerebral circulation: bedside assessment and clinical implications. Crit Care 2016;20:129. 